\title{
DRIL PROCEDURE: TREATMENT FOR VASCULAR ACCESS INDUCED HAND ISCHEMIA. CASE REPORT
}

\begin{abstract}
CLAUDIU HELGIU ${ }^{1}$
I "Lucian Blaga” University of Sibiu

Keywords: vascular Abstract: Native vascular access (AVF arteriovenous fistula) for chronic hemodialysis (CH) access, arteriovenous performed proximally to the diabetic patient, between the brachial artery and the basilic vein or fistula, hemodialysis, cephalic vein, determines the risk of distal (hand) ischemia. The correction of the ischemia can be hand, ischemia done differently depending on its severity, starting from the vasodilator medication, reaching the cancellation of the vascular access with the accomplishment of the chronic hemodialysis on the central venous catheter. DRIL procedure (Distal Revascularization and Interval Ligation) corrects distal ischemia while maintaining functional vascular access, an important aspect in diabetic patients in whom vascular capital is deficient. We further describe a case of a diabetic patient with right L-T brachiocephalic arteriovenous fistula, with important ischemic phenomena in which the DRIL procedure resolved the ischemia, the patient using vascular access as usual, the second postoperative day.
\end{abstract}

\section{INTRODUCTION}

Native vascular access for chronic hemodialysis is the best way to achieve chronic hemodialysis, with a much lower rate of complications (sepsis, thrombosis) than using a central venous catheter or synthetic graft.(1) Ideally, vascular access is performed distally (at the level of the forearm vessels) to the non-dominant limb. The diabetic patient candidate for chronic hemodialysis has a very poor distal vascularity (calcified arteries, phlebitic veins), so that vascular access will have to be achieved proximally (at the level of the arm).(2) The brachial artery is anastomosed with the cephalic vein or the basilic vein at the level of the arm (3), this type of vascular access having an important risk of developing distal ischemia of the hand through vascular steal at the level of arteriovenous anastomosis in systole and diastole.

Depending on the severity of the ischemic symptoms, vascular access induced hand ischemia (VAIHI) is classified in:(4)

- $\quad$ Grade 0 - no symptoms, no treatment.

- $\quad$ Grade 1 - cold skin on the extremity with AVF with mild symptoms that do not require treatment.

- Grade 2 - intermittent ischemic phenomena that occur during hemodialysis, intermittent claudication that requires treatment depending on severity.

- $\quad$ Grade 3 - ischemic rest pain, necrotic lesions, requiring rapid intervention.

DRIL procedure consists of distal revascularization of the hand through an anastomosed venous graft, proximally to at least $10 \mathrm{~cm}$ cranially to the initial arteriovenous anastomosis, and distally to the brachial artery under the primary anastomosis or to the radial or ulnar artery if they do not present extensive calcifications.

The ligation of the brachial artery is accomplished between the primary and distal anastomosis (interval ligation).(5) Preoperatively, the examination of the vascular network at the level of the upper limb is done by Duplex,
Triplex examination, to which is added the examination of the bilateral internal saphenous vein from the level of the arch vein to the distal $1 / 3$ of the calf. Pulseoximetry is done on both hands symmetrically.

The case we present consist of using DRIL procedure like a solution for correcting ischemic phenomena while maintaining functional vascular access and its usual use for chronic hemodialysis.

\section{CASE REPORT}

Patient aged 60 years old, male, with chronic hemodialysis on right proximal vascular access (right L-T brachiocephalic arteriovenous fistula). His past medical history consists of: diabetes with diabetic retinopathy, hypertension, obesity.

The patient has been performing chronic hemodialysis on the right brachio-cephalic fistula for 2 years. For about 3 months he has been progressively showing ischemic phenomena in the right hand with cold skin and paresthesias in the fingers II - V with pains during hemodialysis (grade 2 of VAIHI). The pulse is not perceptible in the radial and ulnar arteries, but becomes perceptible after compression of the arteriovenous anastomosis.

$\mathrm{Sa} \mathrm{O} 2$ at the right hand is $70-75 \%$ in the fingers II $\mathrm{V}$ and after compression of the arteriovenous anastomosis $\mathrm{Sa} \mathrm{O} 2$ $>$ is higher than $90 \%$.

The ultrasound examination at the level of the right arm highlighted:

Afferent branchial artery with a diameter of $6 \mathrm{~mm}$ with compressible calcified walls, efferent artery with a diameter of $4 \mathrm{~mm}$ with the same appearance. The ulnar artery in the lower $1 / 3$ of the forearm with a diameter of $4 \mathrm{~mm}$, with relatively soft, compressible walls. Radial artery at the same level with a diameter of $2 \mathrm{~mm}$, with calcified walls that cannot be compressed. Brachial artery $10 \mathrm{~cm}$ cranial to AVF have a 6 mm diameter compressible.

${ }^{1}$ Corresponding author: Claudiu Helgiu, Str. Lupeni, Nr. 21, Sibiu, România, E-mail: claudiuhelgiu@ yahoo.com, Phone: +40745 317854 Article received on 24.01.2020 and accepted for publication on 02.03.2020 


\section{CLINICAL ASPECTS}

Right internal saphenous vein with a diameter of $4-6$ $\mathrm{mm}$, supple in the thigh, with a rectilinear trajectory, without varicose dilatations, without ostial reflux. The ultrasound was performed from the groin to the level of the calf. It is completed with the examination of the arterial circulation at the level of the lower limb in order to diagnose a severe arteriopathic process that may contraindicate the harvest of the saphenous vein (long incision).

In the evening before the surgery, the patient is disinfected with betadine in the right upper and lower limb and then he is sterile covered on these surfaces.

The surgery was performed under general anesthesia on the day without hemodialysis and lasted about 3 hours (figure no. 1).

\section{Figure no. 1. Dril procedure}

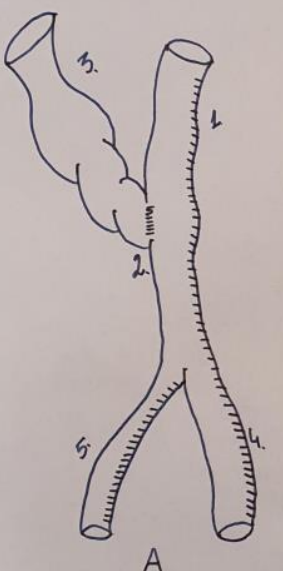

A

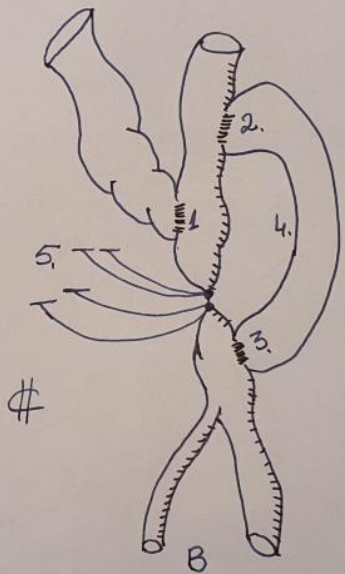

(A) preoperative aspect, 1 brachial artery, 2 arteriovenous anastomosis, 3 cephalic vein, 4 cubita artery, 5 radial artery (B) DRIL, 1 first arteriovenous fistula, 2 second arteriovenous fistula, 3 third arteriovenous fistula, 4 venous graft, 5 brachial ligatures

The surgical field comprises the entire right upper limb, in the abduction position at 90 degrees and the right lower limb along its entire length.

Initially, the right $\mathrm{L}-\mathrm{T}$ brachiocephalic primary anastomosis was detected (primary anastomosis) and the afferent and efferent brachial artery was put on elastic ribbon. Then, the brachial artery is dissected at $10 \mathrm{~cm}$ cranial to the primary anastomosis. After confirming that the proximal brachial artery is suitable, right internal saphenous vein was harvested on a length of $25-26 \mathrm{~cm}$ through a continuous incision in the right thigh, after which the incision is closed by a second surgical team.

The saphenous vein is washed with heparinized serum (12.500 UI Unfractionated heparin per $500 \mathrm{ml}$ physiological saline), dilated and inverted. Cranial anastomosis is performed between the brachial artery and the inverted saphenous graft (secondary anastomosis) in a latero-terminal manner on a diameter of $6 \mathrm{~mm}$ with Premilene suture 7 - 0. The tunneling of the vein on the inner face of the arm is performed, so that the graft does not intersect the basilic vein (for a future fistula between the brachial artery and the basilic vein). The distal anastomosis (tertiary anastomosis) is performed at $3 \mathrm{~cm}$ distally from the primary anastomosis, between the venous graft (terminal) and the brachial artery (lateral) on a diameter of $6 \mathrm{~mm}$ with Premilene suture 7 - 0.

After filling the venous graft with heparined serum, we performed the purge, completed the distal anastomosis (third anastomosi ) and the vein graft was under pressure.

After brachial artery is ligated between the primary and the tertiary anastomosis with Silk no. 3, intraoperative Sa
$\mathrm{O} 2$ after the assembly is finished is over $90 \%$ and fistula was functional with thrill. In the evening of the surgery, the patient has $\mathrm{Sa} \mathrm{O} 2$ over 94\%, without ischemic complaints (figure no 2).

The patient performs the first hemodialysis session 24 hours postoperatively, without ischemic phenomena. He is discharged 72 hours postoperatively. At 6 months postoperatively the assembly was permeable, vascular access was used for chronic hemodialysis and the patient had no ischemic symptoms.

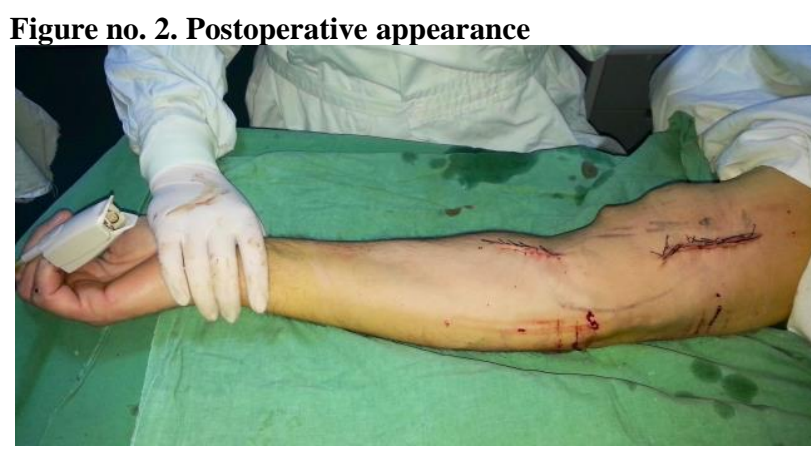

\section{DISCUSSIONS}

Achieving vascular access for proximal chronic hemodialysis through anastomosis between the brachial artery and the cephalic or basilic vein leads to steal phenomenon where, in the systole, part of the blood flow to the hand is diverted in the area with minimal resistance (anastomosed veins).(6) During diastole, blood from the distal territory (forearm, hand) is aspirated at the level of arteriovenous anastomosis, in this way the steal phenomenon was present in both systole and diastole, leading to insufficient arterial flow in the hand and ischemia or necrosis. Ischemic pain is continuous when resting and was accentuated during the hemodialysis session, requiring cessation of hemodialysis.(7)

The surgery indication is given by the presence of rest pain, gangrene of the fingers and the hand. The arteriovenous fistula is usually closed by ligating the vein immediately in the postanastomotic segment. The surgery can be performed under local anesthesia and it restores the distal flow. The disadvantage is that the native vascular access is compromised, and the patient will need to perform HD on CVC, with the decrease of the patient's lifetime. Banding of the vein can be useful, maintaining the vascular access.(8)

After clinical and ultrasound vascular evaluation of arterial and venous capital at the right upper limb and of the saphenous vein we decided to perform a DRIL procedure. Why? Because we can correct the hand ischemia and preserve the vascular access in the same time. The patient despite his diabetes did not have a severe arteriopathy of inferior limb, so we considered that the long tigh incision will heal.

The DRIL procedure was performed for first time in 1988 and reported by Schanzer et al. and it has the advantage of allowing the correction of ischemic manifestations and the functional preservation of vascular access at the same time. $(9,10)$

\section{CONCLUSIONS}

The use of the DRIL procedure to correct distal and secondary arterial flow of ischemic phenomena in the hand in diabetic patients with HD on proximal AVF is a noteworthy solution that improves the arterial flow in the hand and preserves native vascular access for chronic hemodialysis. However, the cases must be carefully selected in terms of arterial pathology in the upper limb (extensive diffuse mediocalcosis that does not allow clamping of the arteries) and 
in the lower limb (severe arteriopathy with distal lesions). The duration of the surgery can be shortened if there are two surgical teams simultaneously.

\section{REFERENCES}

1. Ballard JL, Blunt TJ, Malone JM. Major complications of angioaccess Surgery. Am J Surg. 1992;164:229-232.

2. Sidawy AN, Spergel LM, Besarab A, et al. The Society for Vascular Surgery: Clinical practice guidelines for the surgical placement and maintenance on arteriovenous fistula hemodialysis access. J Vasc Surg. 2008;48(suppl 5):25-255.

3. Nazzal MM. The brachiocephalic fistula: a succesfull secondary vascular access procedure. Vasa. 1990;19:326329.

4. Al Hassanein and Samuel E Wilson. Dialysis Access Associated Ischemic Steal Syndrome. Samuel Eric Wilson Vascular Access Principles and Practice fifth edition. Wolters Kluwer and Lippincott Wiliams and Wilkins Phyladelphia; 2010. p. 178-181.

5. Schanzer H. Overview of complications and management after vascular access creation. In: Gray RJ, ed. Dialysis Access. Philadelphia: Lippincot Williams and Wilkins; 2002. p. 93-97.

6. Holman E, Taylor G. Problems in the Dynamics of blood flow. II. Pressure relations at the site of an arteriovenous fistula. Angiology. 1952;3:415-430.

7. Ingebrigtsen R, Wehn PS. Local blood pressure and direction of flow in experimental arteriovenous fistulae. Acta Chir Scan. 1960;120:142-150.

8. Frank van Hoek, Marc Scheltinga, Martin Luirink, Huub Pasmans, Charles Beerenhouts. Banding of Hemodialysis Access to Treat Hand Ischemia or Cardiac Overload. Seminars in Dialyse. 2009;22(2):204-208.

9. Huber TS, Brown MP, Seeger JM, Lee WA. Midterm outcome after the Distal revascularization and interval ligation (DRIL) procedure. J Vasc Surg. 2008;48(4):926232

10. Knox RC, Berman SS, Hughes JD, Gentile AT, Mills JL. Distal revascularization - interval ligation: a durable and effective treatment for ischemic steal syndrome after hemodialysis access. J Vasc Surg. 2002;36(2):250-255. 